\title{
Little need to incorporate obesity measures in calculations of cardiovascular disease risk
}

A new study published in the Lancet has demonstrated that the commonly used obesity measures BMI, waist circumference, and waist-tohip ratio provide no important additional predictive value to calculations of cardiovascular disease risk that already incorporate information about a patient's blood pressure, lipid levels, and history of diabetes mellitus. Nevertheless, the authors of this new report do point out that their finding "does not, of course, diminish the importance of adiposity as a major modifiable determinant of cardiovascular disease".

David Wormser, an epidemiologist from the University of Cambridge, UK, who was involved in the study explains that "previous observational studies reported conflicting findings regarding the relative importance of overall and abdominal adiposity in relation to risk of cardiovascular disease. Moreover, guidelines have provided differing recommendations about the value of inclusion of clinical measures of adiposity in cardiovascular risk scores." The Emerging Risk Factors Collaboration, therefore, set out to assess the utility of incorporating BMI, waist circumference, and/or waist-to-hip ratio into risk calculations for cardiovascular disease.

Data for 221,934 patients who participated in any one of 58 prospective studies performed in developed countries throughout Europe, North America, and the Asia-Pacific region were assessed by the investigators. All patients had no known history of cardiovascular disease at their study's baseline.

The investigators recorded 14,297 firstever cardiovascular disease outcomes, including 8,290 coronary heart disease outcomes $(4,982$ nonfatal myocardial infarctions and 3,308 deaths attributed to coronary heart disease), 2,906 incident ischemic strokes, 596 hemorrhagic strokes, 2,070 unclassified strokes, and 435

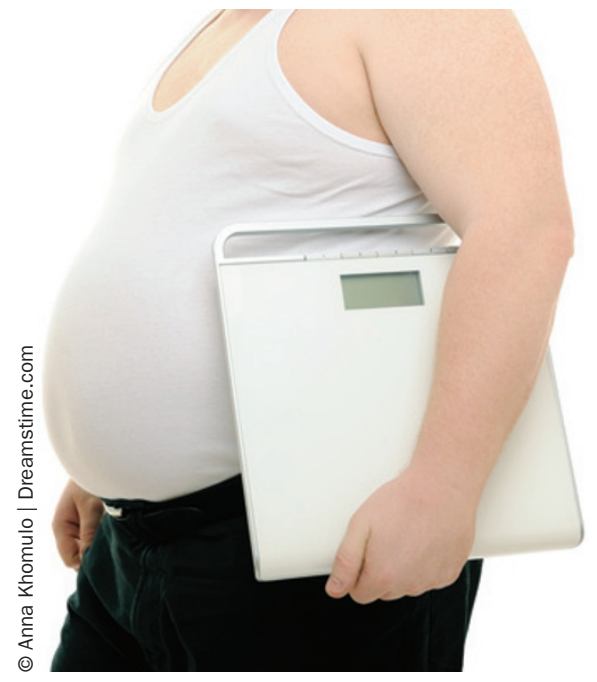

from 0.0006 to 0.0023 versus 0.0087 $\mathrm{C}$-index change). Furthermore, when these obesity measures were added to a cardiovascular disease risk prediction model that included information on all the aforementioned Framingham risk score covariates including levels of total and HDL cholesterol, no obvious effects on cardiovascular disease risk discrimination (C-index changes ranging from -0.0001 to 0.0008 ) or reclassification in predicted 10 -year risk categories (net reclassification improvement ranging from $-0.19 \%$ to $-0.03 \%$ ) were recorded.

"This study underlines the value of general practitioners continuing to measure cholesterol and blood pressure for cardiovascular risk prediction, irrespective of body shape," comments David Wormser. Indeed, in their study report, the investigators went one step further to highlight that, on the basis of the larger impact of blood lipid measures compared with obesity measures on cardiovascular disease risk prediction, there is a "desirability of supporting the development of lipid assessment in resource-poor settings".

Although the study findings point to a lack of utility of obesity measures in cardiovascular disease risk prediction when information on the patient's blood pressure, lipid levels, and diabetes history is available, the authors are quick to point out that "because excess adiposity is a major determinant of the intermediate risk factors noted above, our findings underscore the importance of controlling adiposity to help prevent cardiovascular disease".

Bryony M. Mearns 